\title{
Meteor head echo altitude distributions and the height cutoff effect studied with the EISCAT HPLA UHF and VHF radars
}

\author{
A. Westman ${ }^{1}$, G. Wannberg ${ }^{1}$, and A. Pellinen-Wannberg ${ }^{2}$ \\ ${ }^{1}$ EISCAT Scientific Association, Kiruna, Sweden \\ ${ }^{2}$ Swedish Institute of Space Physics, Kiruna, Sweden
}

Received: 26 February 2003 - Revised: 18 November 2003 - Accepted: 3 February 2004 - Published: 8 April 2004

\begin{abstract}
Meteor head echo altitude distributions have been derived from data collected with the EISCAT VHF $(224 \mathrm{MHz})$ and UHF $(930 \mathrm{MHz})$ high-power, large-aperture (HPLA) radars. At the high-altitude end, the distributions cut off abruptly in a manner reminiscent of the trail echo height ceiling effect observed with classical meteor radars. The target dimensions are shown to be much smaller than both the VHF and the UHF probing wavelengths, but the cutoff heights for the two systems are still clearly different, the VHF cutoff being located several $\mathrm{km}$ above the UHF one. A single-collision meteor-atmosphere interaction model is used to demonstrate that meteors in the (1.3-7.2) $\mu \mathrm{g}$ mass range will ionise such that critical electron density at $224 \mathrm{MHz}$ is first reached at or around the VHF cutoff altitude and critical density at $930 \mathrm{MHz}$ will be reached at the UHF cutoff altitude. The observed seasonal variation in the cutoff altitudes is shown to be a function of the seasonal variation of atmospheric density with altitude. Assuming that the electron density required for detection is in the order of the critical density, the abrupt altitude cutoffs can be explained as a consequence of the micrometeoroid joint size-speed distribution dropping off so fast at the large-mass, high-velocity end that above a certain altitude the number of detectable events becomes vanishingly small. Conversely, meteors at the lowmass end of the distribution will be gradually retarded such that the ionisation they generate never reaches critical density. These particles will remain unobservable.
\end{abstract}

Key words. Radio science (instruments and techniques) Interplatery physics (interplanetary dust) - General or miscellaneous (new fields)

Correspondence to: A. Westman

(assar@eiscat.com)

\section{Introduction}

Meteors have been systematically studied with radars since the late 1940's. Most of the returns detected by conventional meteor radars have scattered from underdense meteor trails, formed in the wake of the meteoroids entering the atmosphere. The altitude range over which such scattering can be observed is quite limited, extending at most from 70 to $140 \mathrm{~km}$ (Steel and Elford, 1991). Studies of the echo altitude distributions show that over a given restricted height range, the maximum detection height is approximataly logarithmically proportional to the probing wavelength (McKinley, 1961). This is known as the meteor height ceiling effect. The effect is assumed to be a consequence of the fact that ionized meteor trails have a finite radial extent. Immediately behind the meteoroid, the ionization rapidly fills a column of radius $r_{0}$, the so-called initial radius. $r_{0}$ is inversely proportional to the neutral atmospheric density and has often been estimated to be equal to about three mean free paths (Manning, 1958). When $r_{0}$ is small compared to the probing wavelength, waves scattered from the front and back of a trail add in phase and reinforce the radar return. To be observable at all by radar the trail must be almost perpendicular to the radar beam. When $r_{0}$ approaches a quarter wavelength, destructive interference between signals returned from different parts of the trail results and the radar echo is strongly attenuated. A meteor radar operating at $6 \mathrm{~m}$ wavelength therefore exhibits a maximum detection altitude of about $105-110 \mathrm{~km}$. Because of the height cutoff effect, VHF radars detect only a fraction of the meteors that should otherwise be observable. One estimate puts the fraction of meteors actually detected at 3-4\% (Olsson-Steel and Elford, 1987). The height ceiling effect also puts an upper limit on the useful frequency, and so most classical meteor radars have operated below $100 \mathrm{MHz}$.

Recent work using incoherent scatter radar facilities - also known as High Power Large Aperture (HPLA) radars in the present context - has identified a new class of meteor radar events, very different from conventional trail echoes. Operating in the $40-1300 \mathrm{MHz}$ range, these highly sensitive 


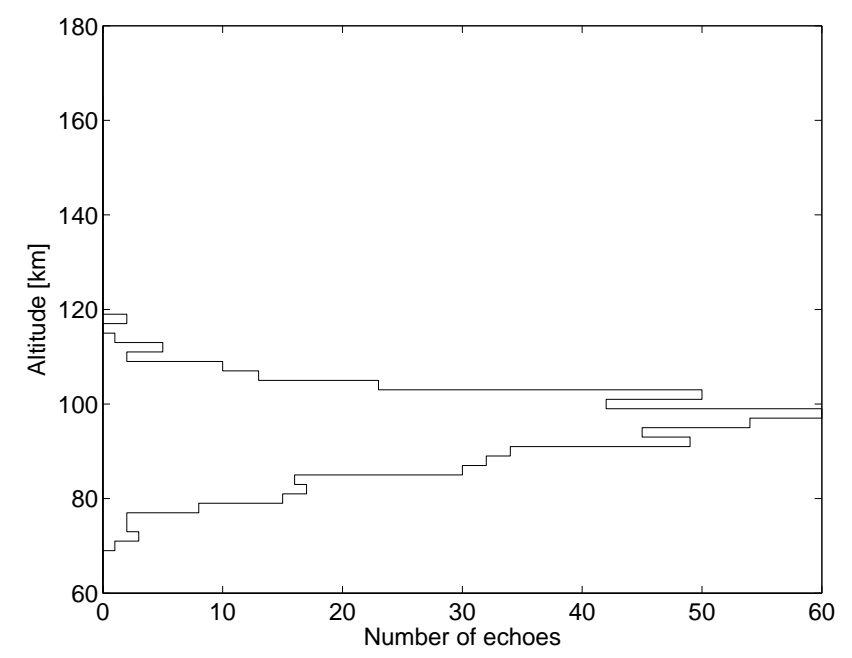

Fig. 1. Altitude distribution of the head echoes observed during the December campaigns 1990 and 1991.

systems regularly observe transient, strongly Doppler shifted and largely aspect insensitive meteor echoes which also exhibit altitude distributions with sharp upper cutoffs, reminiscent of the trail echo height ceiling effect. The scattering mechanism responsible for these echoes is likely to be fundamentally different from the classical trail echo mechanism. HPLA meteor head echo altitude distributions have been recorded by the EISCAT, Arecibo, ALTAIR and Millstone Hill radar systems (Pellinen-Wannberg and Wannberg, 1994; Zhou and Kelley, 1997; Janches et al., 2000; Hunt et al., 2001; Erickson et al., 2001).

The present paper is based on a set of dual frequency EISCAT data from a series of experiments where the $931 \mathrm{MHz}$ (UHF) and $224 \mathrm{MHz}$ (VHF) radar systems are used simultaneously to look for meteors in a common volume in the E-region. We will describe the observational evidence for a head echo cutoff altitude frequency dependence and discuss the effect in terms of a qualitative meteoroid-atmosphere interaction model.

\section{Measurements and data}

The observations discussed in this paper were performed in three sessions, viz in December 1990 before, during and after; in December 1991 before and during the Geminids, and in August 1993 during the Perseids. However, none of the data sets show any signs of shower enhanced meteor rates.

In the December sessions, $66 \mathrm{~h}$ of UHF data were collected, while the VHF radar was run only for $1.25 \mathrm{~h}$. In the August sessions $11.5 \mathrm{~h}$ of UHF and $10.5 \mathrm{~h}$ of VHF data were collected. An overview description of the experiments is given in Pellinen-Wannberg and Wannberg (1994), an indepth discussion of the transient echo observations and the estimation of Doppler velocities and cross sections can be found in Wannberg et al. (1996), and flux estimates based on these data sets are presented in Pellinen-Wannberg et al.

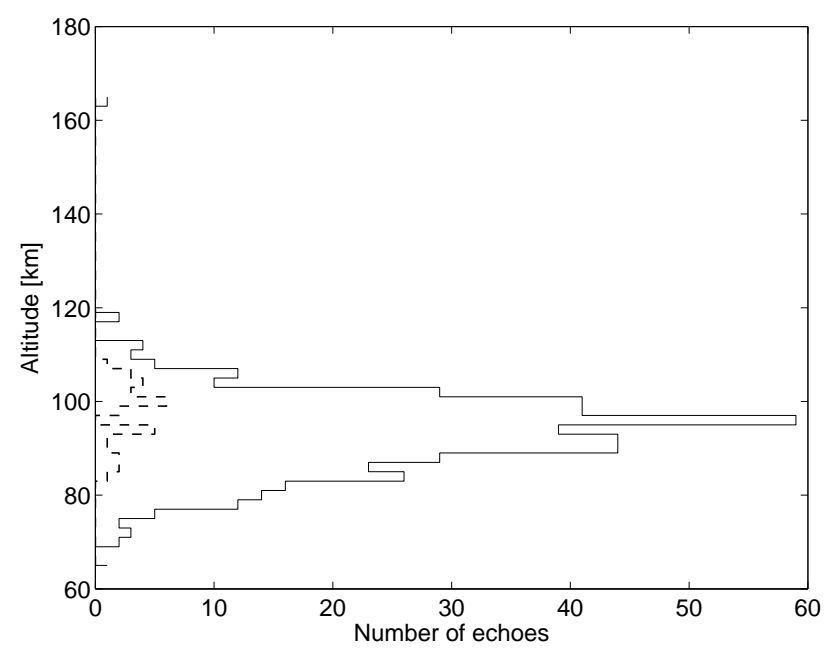

Fig. 2. Altitude distribution of head echoes from the December campaigns recorded with the UHF radar (solid line) and by the VHF radar (dashed line).

(1998). The radar antennas were pointed vertically or close to vertical in all experiments. As the very narrow main beams cover a very small fraction of the visible hemisphere (beam diameters $1-3 \mathrm{~km}$ at meteoric altitudes), in contrast to meteor radars, whose broad beams cover a large solid angle, most of the recorded events are short-lived and represent meteors traversing the beams at arbitrary angles.

The data contains two clearly distinguishable types of echoes. One lasts for a few seconds, displays a very small Doppler shift and is believed to originate from dispersed meteor trails. The other type is the strongly Doppler shifted transient echo discussed above. These echoes last for a few tens of milliseconds, at most. The observed range of Doppler velocity components along the beam is from about 10 to almost $60 \mathrm{~km} \mathrm{~s}^{-1}$, i.e. the scattering centers are moving with meteoric velocities. These echoes are therefore formally similar to the "head-echoes" occasionally observed by conventional meteor radars (Hey et al., 1947; Jones and Webster, 1991).

Figure 1 shows the altitude distribution of all December UHF head echo data. It was clear already from the very first observation that the head echo distribution (based on a sample of 509 echoes) exhibited a fairly abrupt cutoff at about $108 \mathrm{~km}$. Although this observation was made in a different scattering geometry and at a much shorter wavelength than that used by conventional VHF meteor radars, the cutoff was strikingly similar to the classical meteor radar height ceiling effect. As the EISCAT radars are fairly accurately calibrated, we were able to determine the radar cross sections of the transient echo targets to an inaccuracy of about $\pm 10 \%$ (Wannberg et al., 1996). Many echoes from altitudes above $100 \mathrm{~km}$ were found to correspond to apparent target sizes much smaller than the initial radius of a meteor trail, $r_{0}$. Therefore, destructive interference should not have been an issue, but the echoes disappeared quite abruptly nevertheless. 
Table 1. Meteor statistics.

\begin{tabular}{lccccc}
\hline & $\begin{array}{c}\text { data } \\
{[\mathrm{h}]}\end{array}$ & trail echoes & head echoes & $\begin{array}{c}\text { cutoff height } \\
{[\mathrm{km}]}\end{array}$ & $\begin{array}{c}\text { mean free path } \\
{[\mathrm{m}]}\end{array}$ \\
\hline Dec. 1990/91 UHF & 66 & 184 & 509 & $108.0 \pm 0.3$ & 0.5 \\
\hline Dec. 1990/91 VHF & 1.25 & 74 & 31 & $112.0 \pm 1.2$ & 1.1 \\
\hline Aug. 1993 UHF & 11.5 & - & 105 & $104.0 \pm 0.2$ & 0.3 \\
\hline Aug. 1993 VHF & 10.5 & - & 795 & $109.8 \pm 0.3$ & 0.8 \\
\hline
\end{tabular}

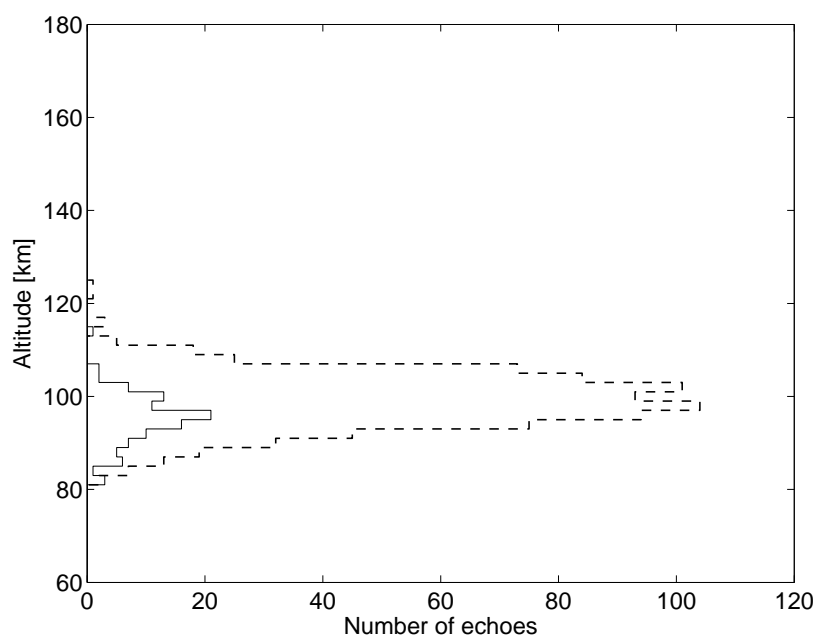

Fig. 3. Altitude distribution of head echoes from the August campaign 1993 recorded with the UHF radar (solid line) and by the VHF radar (dashed line). Note the difference in rate scale from the previous figure.

So, even though there was some process leading to the appearance of an observation "ceiling" for these transient radar returns, the underlying mechanism was unlikely to be the same as the one causing the classical trail echo ceiling.

When the 1990 measurement campaign was planned, the primary scientific goal was the possible detection and mass analysis of meteor-induced $\mathrm{E}_{S}$-layers. It was not expected that using the VHF radar would add any significant information in this application, so it was not used for regular measurements. However, it was turned on for test purposes for a short time, and during this brief operation 31 head echoes were registered. In Fig. 2, the VHF and the UHF head echo data are plotted together. Even though the VHF sample is very small, the VHF height distribution appears to be centered at a slightly higher altitude than the UHF one.

This observation prompted us to run both radars for the duration of the 1993 August campaign. Figure 3 shows all head echo data from this session. 10.5 h of VHF data now contain about 7-8 times as many echoes as $11.5 \mathrm{~h}$ of UHF data, and the VHF distribution displays a distinct upper cutoff, which clearly occurs at a higher altitude than the corresponding UHF cutoff.

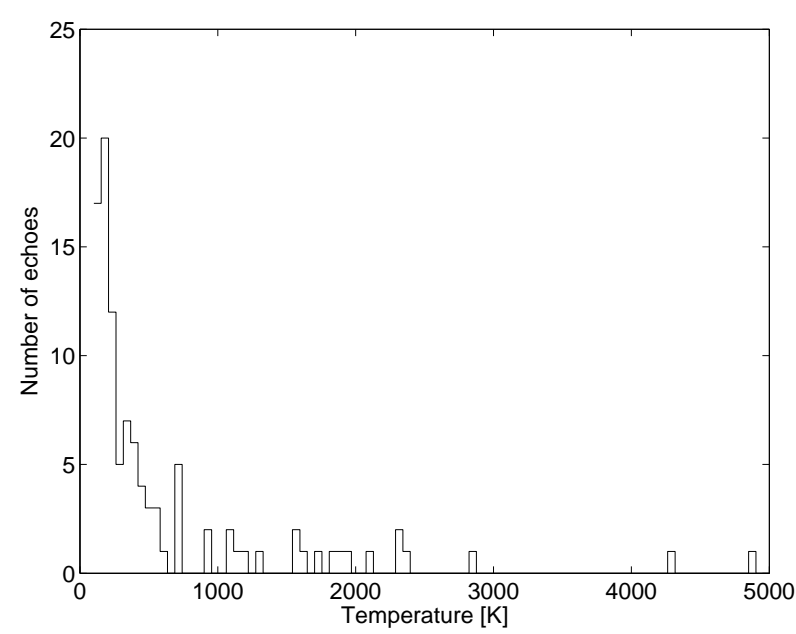

(a)

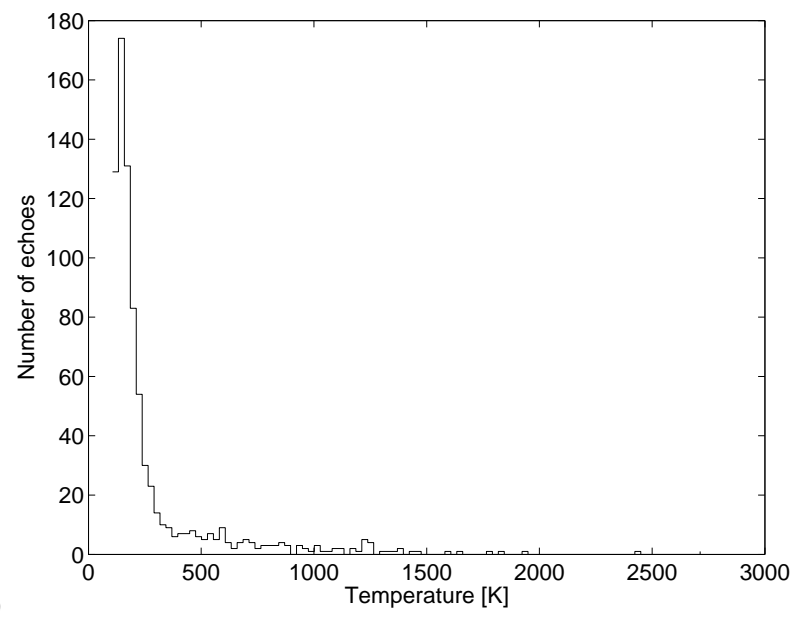

(b)

Fig. 4. All UHF (a) and VHF (b) head echoes as a function of the event equivalent noise temperature. The $3 \sigma$ threshold values are $30 \mathrm{~K}$ for the UHF and $90 \mathrm{~K}$ for the VHF.

In order to compare the altitude distributions, we have, for each session and radar frequency separately, estimated the " $90 \%$-altitudes", i.e. those altitudes below which $90 \%$ of the head echoes are observed. The $90 \%$-altitudes will hereafter be referred to as the "cutoff heights". Echo statistics and cutoff heights for the different measurement periods are summarized in Table 1. 


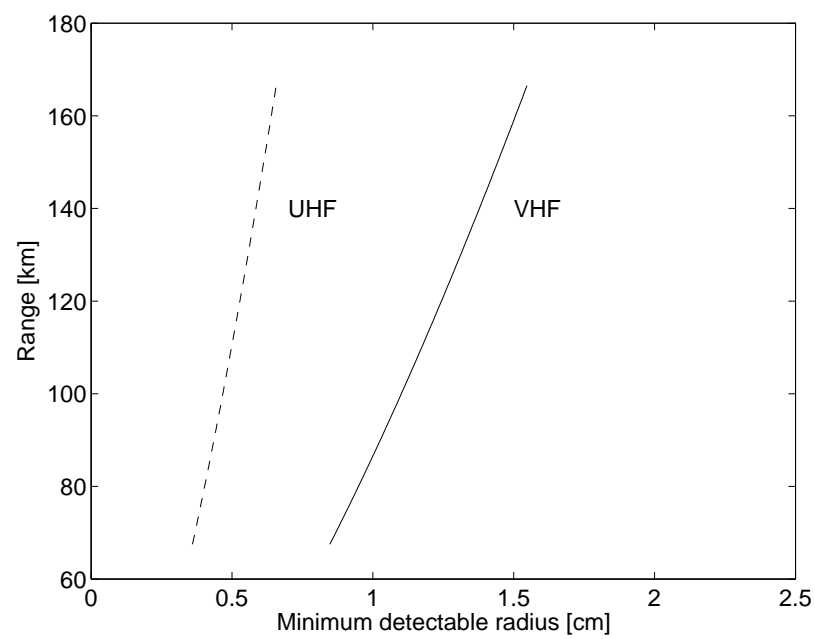

Fig. 5. Overdense plasma target $3 \sigma$ detectability limit, assuming Rayleigh scattering and EISCAT radar parameters.

The error bars here are determined with standard error analysis. To check the low statistics December VHF data, $25 \%$ of the data was removed in a random fashion and the $90 \%$ cutoff height was calculated for 100 cases. This resulted in a standard deviation of $1.2 \mathrm{~km}$.

Radars are noise-limited sensors and the system baselines therefore always exhibit a statistical variance $\sigma$, basically set by the total system noise temperature and the observation bandwidth. In the present study, only echoes rising more than $3 \sigma$ above the average baselines are accepted as unambiguous events. The selection procedures are described in more detail in Pellinen-Wannberg and Wannberg (1994). Using this relatively high detection threshold, the false-alarm rate is minimized, but, on the other hand, there is a risk that a significant fraction of genuine, but weak, events are excluded. To see if this is the case or not, all unambiguous UHF and VHF head echoes have been sorted according to equivalent signal temperature (Wannberg et al., 1996). The resulting distributions, averaged over all altitudes, are shown in Fig. 4a and Fig. 4b. It is seen that most of the echoes occur at around $150 \mathrm{~K}$ and that the occurrence rates drop rapidly with increasing signal temperature. But the rates also drop for signal temperatures below $150 \mathrm{~K}$, indicating that the $3 \sigma$ threshold does indeed pass events that are so weak that they fall below the peak of the distribution function. This observation will be important for the following discussion.

Before discussing the effect in physical terms, we shall first demonstrate that it is not an artifact of limited equipment sensitivity. To this end, we refer back to our previous papers of Wannberg et al. (1996); Pellinen-Wannberg et al. (1998), in which Rayleigh scattering from compact "balls" of plasma contained within the meteor coma was proposed as a possible scattering mechanism, in qualitative agreement with the observations. The most important numerical results from these papers were that the plasma target radii were estimated to be $2-4 \mathrm{~cm}$ for the VHF and $1-2 \mathrm{~cm}$ for the UHF

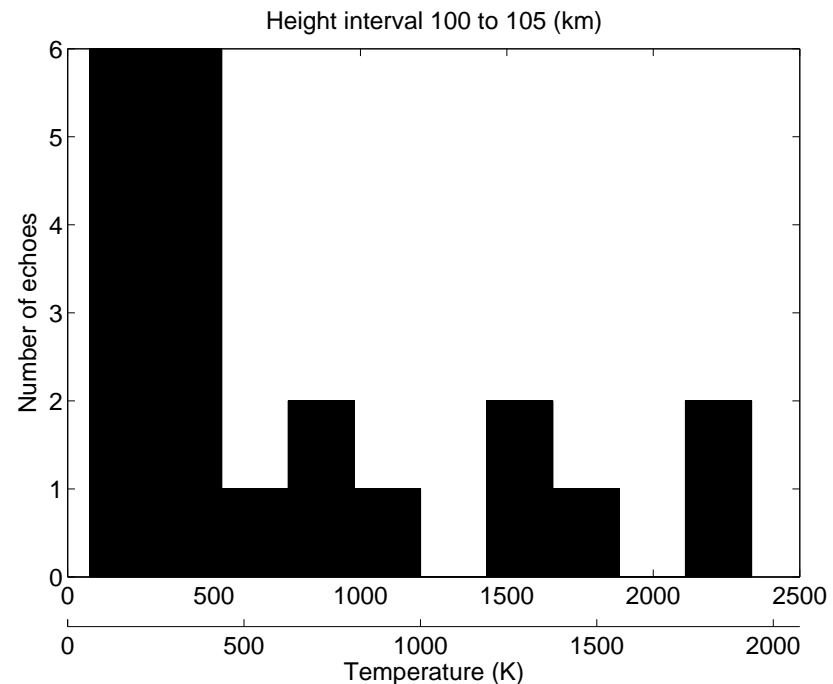

Fig. 6. The effect of the range factor of the scattering from the August UHF target temperature distribution over 100-105 km altitude range (the upper axis). The lower axis gives the equivalent noise temperature as if the same distribution had been displaced $5 \mathrm{~km}$ upwards.

head echoes from $0.1-1.0 \mathrm{~mm}$ meteoroids. Leaving the issue of the actual scattering mechanism aside for the moment, we shall use the Rayleigh scattering model in the following discussion, as it provides a simple and restrictive mechanism for estimating minimum observable target sizes. Figure 5 shows the smallest Rayleigh scatter target radii that can be detected by the EISCAT radars, assuming a $3 \sigma$ detection threshold and 1990 system parameters. The threshold target radius $r$ varies with range $R$ as $r \propto R^{2 / 3}$, and thus it grows only relatively slowly with the altitude. In the Rayleigh picture, the $3 \sigma$ limits, which fall at equivalent target temperatures $30 \mathrm{~K}$ for the UHF and $90 \mathrm{~K}$ for the VHF, correspond to target radii of about $0.3 \times 10^{-2} \mathrm{~m}$ at UHF and $1.1 \times 10^{-2} \mathrm{~m}$ at VHF, respectively, assuming that the targets are located in the center of the antenna beams. These target sizes are well above the detection limits of the radars, up to altitudes of more than $180 \mathrm{~km}$. On the other hand, the upper cutoffs of the statistical head echo distributions are very sharp, and they all occur in the height range where the classical trail echo ceiling effect is observed. The computed cutoff heights from Table 1 are $108.0 \pm 0.3 \mathrm{~km}$ for the UHF and $112.0 \pm 1.2 \mathrm{~km}$ for the VHF December data, and $104.0 \pm 0.2 \mathrm{~km}$ for the UHF and $109.8 \pm 0.3 \mathrm{~km}$ for the VHF August data, respectively. Thus, insufficient system sensitivity does not appear to be an issue.

To further demonstrate that apparatus effects are not affecting the observed cutoff heights, the distributions close to cutoff have been studied in more detail. Figure 6 shows the UHF target temperature distribution for the August observation over the $100-105 \mathrm{~km}$ altitude range, which includes the cutoff height at $104 \mathrm{~km}$. This figure also includes a second abscissa, which is scaled as if the physical target size distribution had been displaced upwards in altitude by $5 \mathrm{~km}$, i.e. to $105-110 \mathrm{~km}$. By inspection, it is seen that such a 
translation would cut out only a small fraction of the echoes and leave most of the distribution substantially untouched and detectable - but the observed cutoff is much more abrupt.

In principle, a radar echo directly off a solid meteoroid body should show the same Doppler characteristics as those exhibited by our observed events. However, if it is assumed that the effective radar cross sections of meteoroids are similar to those of comparably-sized Rayleigh scatterers, it follows that only centimeter-size or larger meteoroids would be directly detectable by the EISCAT radars in the $100-180 \mathrm{~km}$ range. The available meteor statistics predicts that only about $0.1 \%$ of all meteors are of this size, or larger (Hughes, 1978), so that in a total sample of some 1400 events maybe 1 or 2 are big enough to produce an echo from the meteoroid body itself. In fact, no head echoes at all were detected beyond about $125 \mathrm{~km}$.

Three important conclusions can be drawn: very few, or none, of the observed echoes are from solid meteoroids, the targets that we observe are therefore necessarily plasma targets, and - most importantly - these form only below some critical altitude, which is, however, different for the two radars and different at different times of the year.

\section{Experimental bias}

As the antenna beam widths of the EISCAT VHF and UHF systems are unequal, the observed event altitudes contain beamwidth-related terms that are different for the two systems. We now investigate how much the cutoff altitude difference estimate is biased as a result.

The UHF antenna $-3 \mathrm{~dB}$ beamwidth is $0.6^{\circ}$, corresponding to a $1 \mathrm{~km}$ half-power beam diameter at $100 \mathrm{~km}$ range. The VHF system uses a $120 \mathrm{~m} \times 40 \mathrm{~m}$ parabolic cylinder antenna, which produces a roughly elliptical $1.2^{\circ} \times 1.7^{\circ}-3 \mathrm{~dB}$ beam, corresponding to a $2 \mathrm{~km}$ by $3 \mathrm{~km}$ half-power beamwidth at $100 \mathrm{~km}$ range.

The two antennas are co-located, the distance between the phase centers being about $150 \mathrm{~m}$. The UHF beam is thus completely enclosed by the VHF beam at $100 \mathrm{~km}$ altitude when both are pointed vertically. A meteor entering the atmosphere in a plane through both beams will first cross the $-3 \mathrm{~dB}$ boundary of the VHF beam. Some tens of milliseconds later it enters the UHF beam at a slightly lower altitude. The altitude difference, $h$, is:

$h=\frac{H_{V}}{d_{V}}\left[D+\frac{d_{V}-d_{U}}{2}\right]$,

where $d_{V}$ and $d_{U}$ are the $-3 \mathrm{~dB}$ main beam diameters of the VHF and the UHF systems, $D$ is the separation between the antennas and $H_{V}$ is the observed altitude extent of the VHF event. Inserting numerical values (using both the minimum and the maximum value for $d_{V}$ ) gives

$h=(0.22 \ldots 0.38) H_{V}$,

where $h$ is in kilometers.
The effect could be even larger if targets were first being detected in the sidelobes of the VHF antenna, whose sidelobe suppression is not brilliant. The two-way suppression through the first sidelobe is only $-26 \mathrm{~dB}$, and so some of our weakest echoes could actually have been picked up through it. However, as the outer edge of the first sidelobe is offset from the main lobe by about $3.4^{\circ}$, the resulting altitude bias is at most some $200 \mathrm{~m}$. Pickup through the third and higher sidelobes at levels exceeding the $3 \sigma$ limit would be extremely rare and is not considered to be a problem.

The degree to which the altitude data becomes biased is a function of the data taking mode. Technical limitations of the data processing system in use at EISCAT at the time forced us to record the December data set in a time-averaging mode with a time resolution of $2 \mathrm{~s}$ and an altitude resolution of $450 \mathrm{~m}$. In this mode, meteor events in general show up only in a single 2-s time bin, containing the sum of all radar returns from the target as it crosses the beam. This composite echo is dominated by returns from the time of closest approach, i.e. when the target is closest to the beam center. In this case the best-fit altitude values from both systems are directly comparable and the beamwidth bias does not enter into the picture.

The August 1993 data was recorded with both beams directed to zenith to within $0.1^{\circ}$ and with a 6 -ms time resolution. Every one of the 105 recorded UHF events was accompanied by a simultaenous detection by the VHF radar. About 50 of these dual events could be analysed for velocity; an example of a such an event can be found in Wannberg et al. (1996). For this data set, the altitude of an event is defined as the first altitude of detection, which should cause the bias to show up in full. None of the observed events extends for more than $2 \mathrm{~km}$ along the VHF line-of-sight, leading to a maximum value for $h=0.76 \mathrm{~km}$. Assuming that the observed targets represent a random distribution of orbits, entering the asymmetric VHF beam from all directions, the average bias becomes smaller, of the order of $0.58 \mathrm{~km}$, or less than $10 \%$ of the observed $6 \mathrm{~km}$ difference.

We conclude that the observed differences in the altitude distributions are real and not dominated by beamwidthdependent biases. Very similar frequency-dependent altitude distributions can be found in recent data from the ALTAIR radar (Hunt et al., 2001), although those authors did not specifically discuss the altitude distributions.

\section{The meteor impact mechanism and the head echo}

On its way through the atmosphere, a meteoroid passes through a sequence of different interaction regimes, characterized by very different, and partially highly complex, gas and plasma dynamics. However, at upper altitudes where the atmospheric density is still so low that the meteoroid is much smaller than $l_{\infty}$, the atmospheric mean free path, defined by

$l_{\infty}=\frac{1}{\sqrt{2} n_{0} \sigma_{0}}$, 


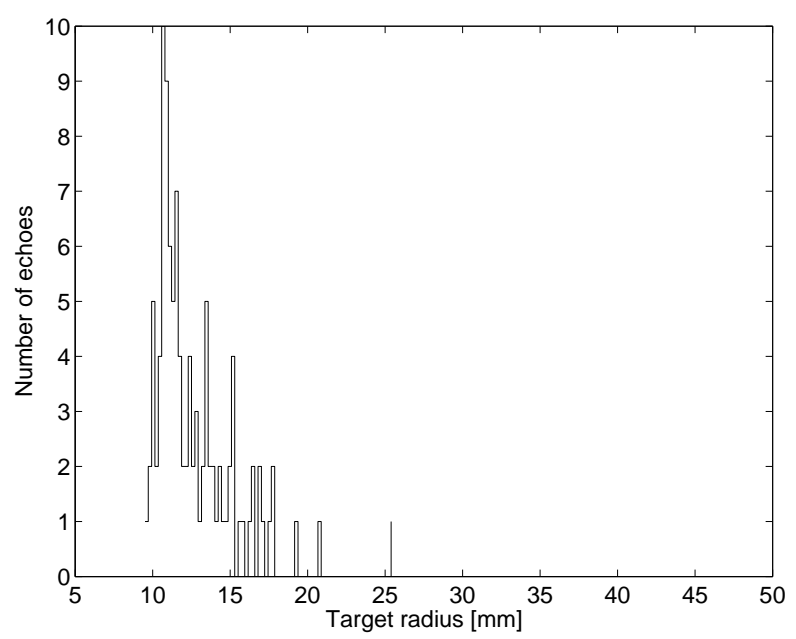

(a)

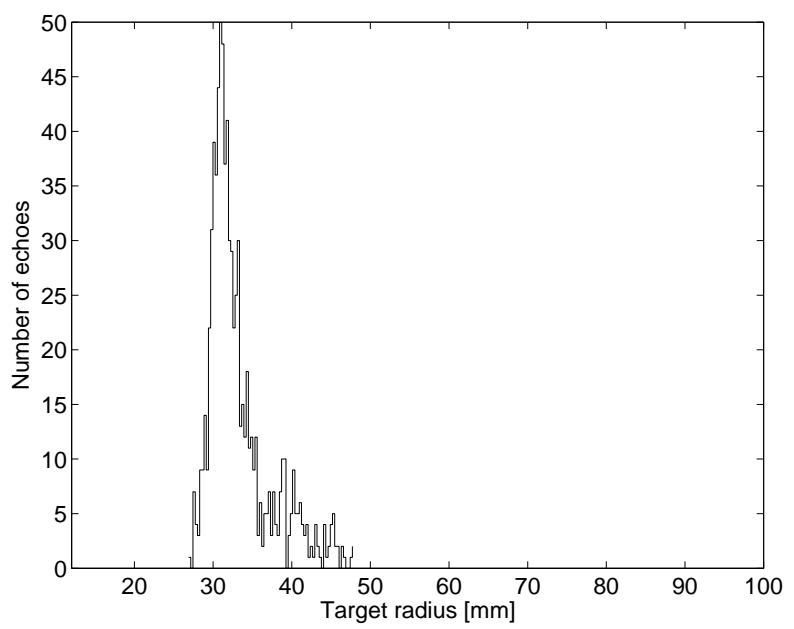

(b)

Fig. 7. All UHF (a) and VHF (b) head echoes as a function of equivalent target radii, assuming that the target behave as Rayleigh scatterers and all of them passed through the beam center.

where $n_{0}$ is the neutral number density and $\sigma_{0}$ is the collision cross section, the particle is in the so-called free-molecular regime (Bronshten, 1983), where the gas dynamics is relatively simple. Here, the primary interaction takes place through single collisions between individual air molecules and the meteoroid; flow and drag effects are insignificant, and the dynamics can be well described by the so-called theory of first collisions.

We have used the MSISE-90 atmosphere model (Hedin, 1991) to estimate the atmospheric number density, $n_{0}$, and the atmospheric mean free path, $l_{\infty}$, for the two observation seasons (mid-December and mid-August). In all cases, the mean free paths at the cutoff heights are $0.4 \mathrm{~m}$ or more, so that the meteoroid-atmosphere interaction is indeed in the free-molecular regime, at least for millimeter-size or smaller meteoroids. For each radar wavelength separately, the mean free paths at the cutoff are found to be approximately the same, even though the heights measured in the December and August sessions differ by $2-4 \mathrm{~km}$. Therefore, the head echo cutoff effect appears to be strongly controlled by col-

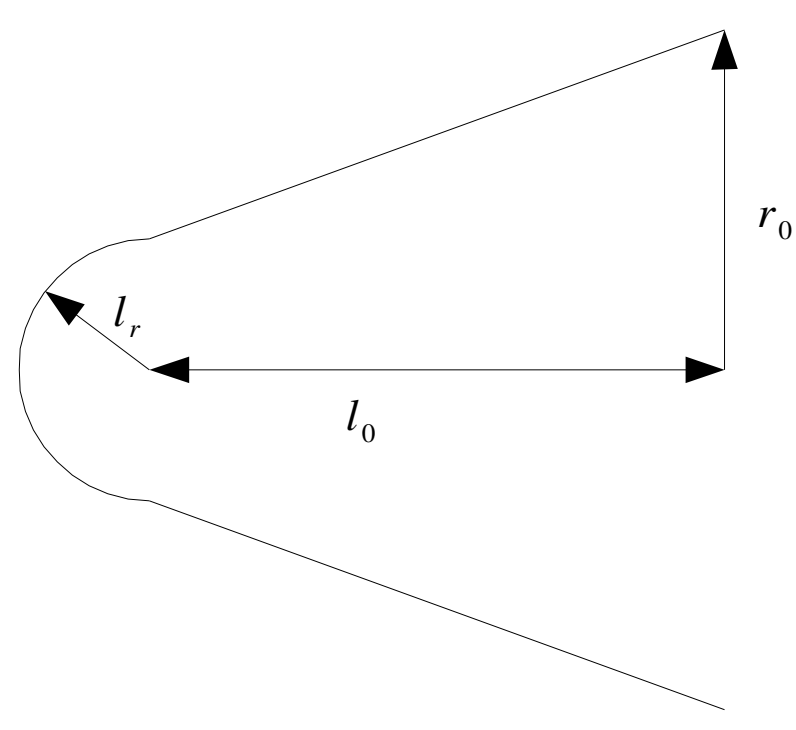

Fig. 8. Simplified view of the region closest to the meteor, where $l_{r}$ is the mean free path length for the scattered and emitted molecules from the meteoroid, $l_{o}$ is the distance the meteoroid moves before a scattered molecule becomes thermalized, and $r_{O}$ is the initial trail radius. Note this drawing is not to scale.

lisional processes governed by atmospheric number density and/or mean free path, but not by altitude as such.

The mean free path at the VHF $(\lambda=1.3 \mathrm{~m})$ cutoff altitude is about $1.1 \mathrm{~m}$, while the mean free path at the $\operatorname{UHF}(\lambda=0.33$ $\mathrm{m}$ ) cutoff altitude is about $0.5 \mathrm{~m}$. In other words, a change in wavelength by a factor of about 4 moves the cutoff altitude upwards to a point where the mean free path has increased by a factor of about 2.2. This shows that the head echo cutoff effect is wavelength dependent, but the relationship between probing wavelength and mean free path at the cutoff is nonlinear, in contrast to the trail echo case.

What remains is to suggest a plausible mechanism that can reproduce both the actually observed head echo cross sections, and the drop in event rate at the cutoff altitude, while taking wavelength and atmospheric density into account. Unfortunately, it is not straightforward to convert the observed source temperatures into equivalent target sizes, since it is impossible to tell from the data how far from the beam axis a particular meteor intersects the radar beam (Wannberg et al., 1996). The effect of this uncertainty is that the sizes of all targets, except those that pass through the exact center of the beam, will always be underestimated to some degree. Keeping this in mind, we refer to Fig. 7a and Fig. $7 \mathrm{~b}$. These are basically the same as the distribution plots of Fig. 4a and Fig. 4b, but now with the abscissas scaled in units of equivalent target radius, assuming that a) all targets have passed through the beam center, and that $b$ ) the targets behave as Rayleigh scatterers. Under these assumptions, the target radii cluster in the ranges 10-17 mm (UHF) and 26$45 \mathrm{~mm}$ (VHF). 


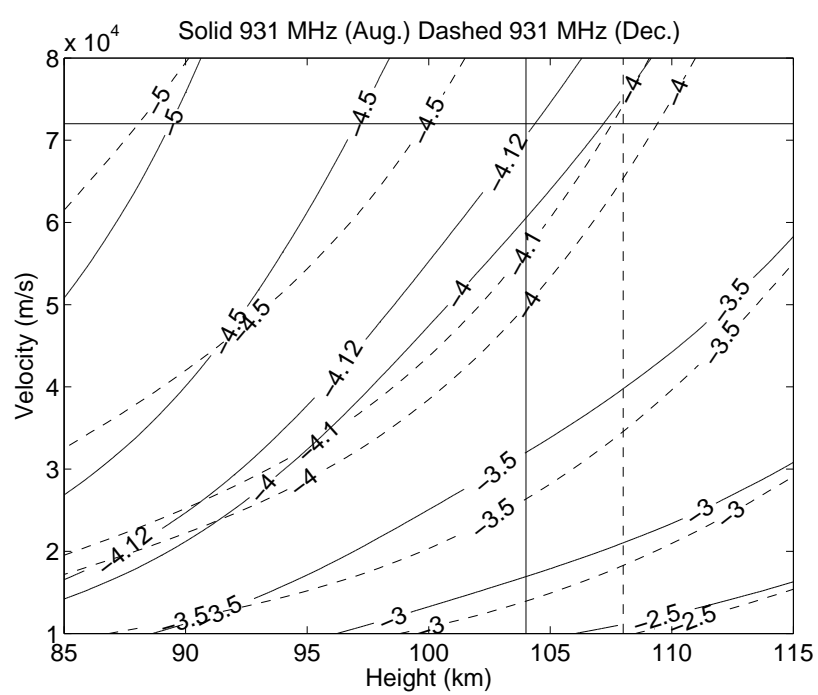

Fig. 9. Showing the altitude at which the ionisation produced by a meteor of a given size first reaches critical density at the EISCAT UHF frequency, $930 \mathrm{MHz}$, with $\log$ (meteor radius in meters) as a parameter. Height is related to atmospheric density through the MSISE-90 model. Solid lines represent an August atmosphere and dashed lines a December atmosphere. The vertical lines indicate the corresponding cutoff altitudes.

A simple physical picture that can potentially explain the production of such small and dense plasma concentrations is illustrated in Fig. 8. In the free-molecular regime, a meteoroid dissipates its kinetic energy through inelastic collisions with air molecules. Since it enters the atmosphere at hypersonic velocity, the collisions all occur on the front side of the meteoroid. For typical meteor velocities and atmospheric molecular masses, the estimated energy transferred per collision is of the order of a few hundred $\mathrm{eV}$, sufficient to evaporate several atoms off the meteoroid surface. The evaporated atoms, which leave the surface in arbitrary directions, are all suprathermal relative to the ambient atmosphere, and several or all of them may ionize in the process. In the frame of reference moving with the meteoroid, the effective mean free path of the evaporated molecules is much shorter than $l_{\infty}$. They move on the average a distance $l_{r}$ before they collide with an air molecule (Bronshten, 1983):

$l_{r}=\frac{v_{r}}{n_{0} v_{\infty} \sigma_{0}}$,

where $v_{r}$ is the velocity of molecules reflected from the meteor body and $v_{\infty}$ is the thermal velocity of the ambient atmospheric molecules. For the case of a large Reynolds number (the ratio of inertial forces to viscous forces in ambient flow), a simplified expression for $l_{r}$ is:

$l_{r} \sim(6.3 / M) l_{\infty}$,

where $M$ is the meteor Mach number and $l_{\infty}$ is the atmospheric mean free path.

Thus, single collisions and/or evaporation create a very dense plasma, filling a hemispherical region of radius $\approx l_{r}$

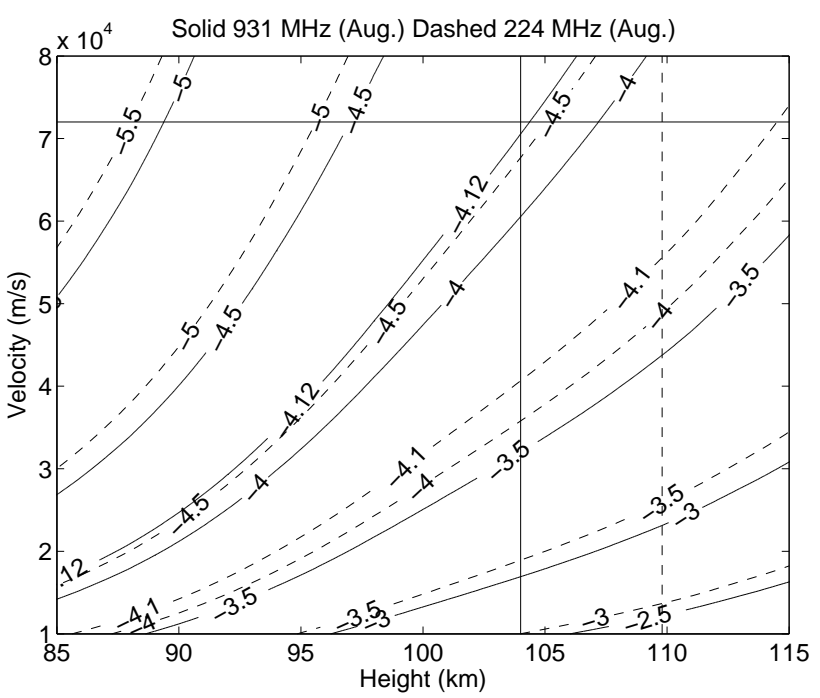

Fig. 10. As Fig. 9, but showing UHF and VHF (224 MHz) critical density altitudes in an August atmosphere. Dashed lines are VHF, solid lines are UHF. The vertical lines indicate the corresponding cutoff altitude.

in front of the meteoroid, and on the average the plasma constituents begin to interact with the neutral air only at this radius. For typical meteoric velocities, $l_{r}$ is of the order of a few tens of centimeters as at $120 \mathrm{~km}$ altitude and about $10 \mathrm{~mm}$ at $104 \mathrm{~km}$ altitude, that is, of the same order as the target sizes estimated above.

The initial radius model (Manning, 1958; Bronshten, 1983), has long been regarded as the standard description of trail formation. Behind the meteoroid, the suprathermal atoms eventually thermalize after a number (8-10) of subsequent collisions with the air molecules. Some of these secondary collisions also produce ionization. When the energy exchange in any collision is no longer sufficient to ionize any of the constituents, ambipolar diffusion takes over and begins to disperse the ionized trail.

At the point where the ionization stops, and before recombination becomes important, the line density $q$, i.e. the number of electrons per unit length of trail, is a conserved quantity. Many workers (Bronshten, 1983; McKinley, 1961) have derived expressions for $q$ as a function of meteor speed, meteor size and atmospheric density. Once an estimate of $q$ is available, a lower bound for the average electron density in the frontal hemisphere can be estimated from:

$d_{o}=\frac{3 q l_{r} f_{1}}{2 \pi l_{r}^{3}}=\frac{3}{2 \pi} q f_{1} l_{r}^{-2}$,

where $f_{1}$ is a dimensionless factor equal to the fraction of the total ionization produced in the primary interactions on the front side of meteoroid, assuming (very conservatively) that the number of electrons liberated per collision is approximately equal for all collisions up to the tenth. This gives $f_{1}=0.1$, which then yields

$d_{o}=5 \times 10^{-2} q / l_{r}^{2}$. 


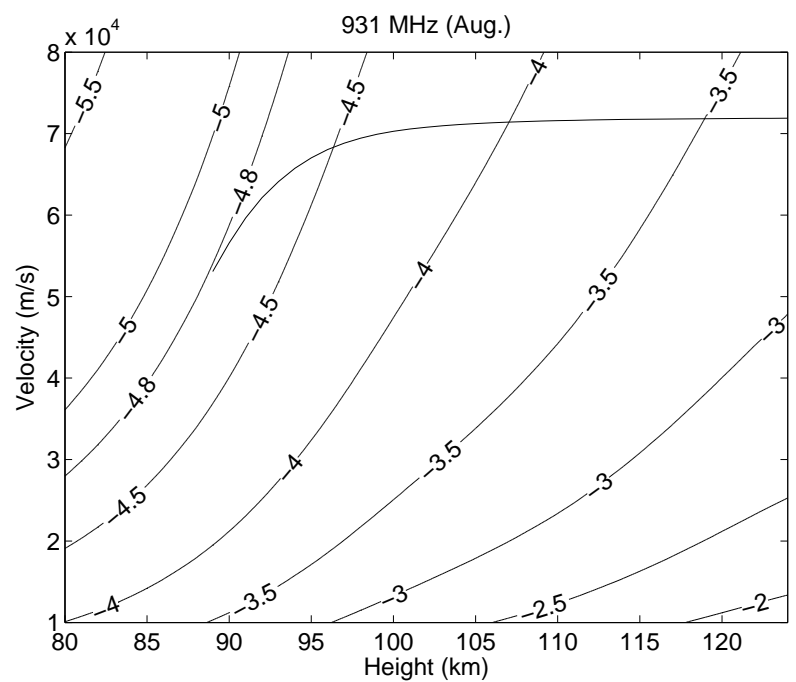

(a)

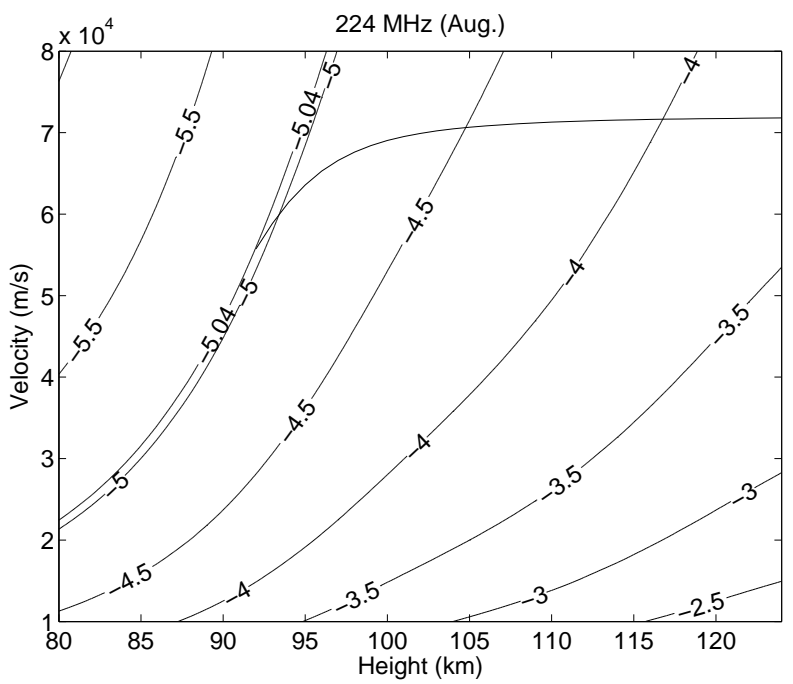

(b)

Fig. 11. Showing how the ionisation produced by $72 \mathrm{~km} \mathrm{~s}^{-1} \mathrm{me}-$ teoroids with $\log (r)$ of -4.8 and -5.04 will approach critical density at one point along their paths at, respectively, $930 \mathrm{MHz}$ (a) and $224 \mathrm{MHz}$ (b), assuming a single collision interaction model. Lower velocity particles of these sizes will not produce critical electron densities at any point. As the single collision model is not valid at lower altitudes, the retardation curves are only computed down to around $90 \mathrm{~km}$ altitude.

Under this assumption, and for typical target radii, $d_{0}$ can become very large indeed, such as $1 \times 10^{16} \mathrm{~m}^{-3}$, the critical density corresponding to a frequency of $931 \mathrm{MHz}$. The proposed mechanism is therefore sufficient to generate the high electron densities that are required to explain our observations.

\section{Discussion}

Our earlier analysis of dual wavelength data (Wannberg et al., 1996) indicates that Rayleigh scatter from overdense plasma could explain the measured cross sections and observed wavelength dependence. Mathews et al. (1997) have suggested a different model, where the head echoes are ascribed to coherent scatter from underdense plasma. However, the Rayleigh scatter hypothesis has recently received strong support from multi-frequency observations at the ALTAIR radars by Close et al. (2002). In the following, we assume that the electron density required for detection is of the order of the critical density but make no other assumptions about the actual scattering mechanism.

Even though the data sets discussed here were collected during two major meteor showers, rate and orbit analyses indicate that the data contain almost no shower meteors. This is in agreement with recent Arecibo results (Janches et al., 2001), so we assume the observed events to be associated with the background meteoroid population.

To see how well the upper parts of the observed altitude distributions are represented by the single-collision interaction model, we have used it to compute, for a range of meteoroid sizes, the height at which the ionisation first reaches critical density as a function of particle speed. The MSISE90 model is used to establish the altitude vs. neutral density relationship as a function of location and time of year. Figure 9 shows the results for $930 \mathrm{MHz}$ (the EISCAT UHF system). There are two sets of curves with the particle radius as a parameter, the full line one representing an August atmosphere and the dashed one representing December conditions. Figure 10 shows UHF and VHF (224 MHz) data from the August 1993 observations in the same format.

It is trivially clear that the smaller the particle, the greater the velocity required to produce critical electron density at a given altitude. Conversely, at a given velocity, the larger the particle, the higher the altitude at which the electron cloud produced by it will first reach critical density. From Fig. 9 we find that at a speed of $72 \mathrm{~km} \mathrm{~s}^{-1}$ (the maximum speed of a particle on a bound orbit as measured in a terrestrial frame of reference), a particle radius of $10^{-4.16} \mathrm{~m}(69 \mu \mathrm{m})$ is sufficient to reach critical density at $930 \mathrm{MHz}$ at the August cutoff height, $104 \mathrm{~km}$. In a December atmosphere at $108 \mathrm{~km}$, a radius of $10^{-4.08} \mathrm{~m}(83 \mu \mathrm{m})$ is required. Similarly, the August 1993 dual frequency data shows that at $109.8 \mathrm{~km}$, the VHF cutoff altitude, a particle radius of $10^{-4.33} \mathrm{~m}(47 \mu \mathrm{m})$ is required to reach critical density at $224 \mathrm{MHz}$.

Choosing $72 \mathrm{~km} \mathrm{~s}^{-1}$ as an upper speed limit for the detected population is not arbitrary. The recent work by Janches et al. (2002) puts the average velocity of a set of 10 tristatic EISCAT UHF meteor events at $64.7 \mathrm{~km} \mathrm{~s}^{-1}$, with eight out of the ten velocities falling in the (56.572.7) $\mathrm{km} \mathrm{s}^{-1}$ interval.

Assuming a canonical density of $3 \times 10^{3} \mathrm{~kg} \mathrm{~m}^{-3}$, particles in the size range discussed above have masses in the (1.3-7.2) $\mu \mathrm{g}$ range, i.e. very close to the peak of the cosmic dust mass flux vs. particle mass distributions derived by other workers (Love and Brownlee, 1993; Ceplecha et al., 1998; Mathews et al., 1997). Around the peak, these distributions are fairly flat, implying that the number flux is inversely proportional to particle mass. In other words, the cumulative number flux is dominated by the low mass particles. For masses greater than about $10 \mu \mathrm{g}$, all distributions 
fall off roughly inversely proportional to particle mass, and so the number fluxes in that region go as (particle mass) ${ }^{-2}$. Thus, the total number of dust particles with masses larger than some $70 \mu \mathrm{g}$ (equivalent to a radius of $10^{-3.15} \mathrm{~m}$ ), which should be capable of producing detectable ionisation at altitudes above the cutoff and up to $130-135 \mathrm{~km}$, is less than $1 \%$ of the total number of particles in the (1.3-7.2) $\mu \mathrm{g}$ range. The number of radar events attributable to these larger particles is likely to be correspondingly small at all altitudes, and particularly so at altitudes above the cutoff, since for them to ionise enough to become detectable, they must also be fast.

Conversely, particles below some critical mass will decelerate gradually in such a way that they never reach a point in velocity-height space where the ionisation approaches critical density. As an example, Fig. 11a shows how $72 \mathrm{~km} \mathrm{~s}^{-1}$ particles of radius $10^{-4.8} \mathrm{~m}$ (corresponding to $0.05 \mu \mathrm{g}$ ) will just fail to achieve critical density at $930 \mathrm{MHz}$ at any altitude. A similar plot for $224 \mathrm{MHz}$ is shown in Fig. $11 \mathrm{~b}$, the limit radius in this case is $10^{-5.04} \mathrm{~m}(0.01 \mu \mathrm{g})$.

The foregoing suggests that, on the assumption that the minimum electron density required for detection is of the order of the critical density at the radar frequency, both the observed cutoff altitude effect and its variation with radar wavelength follow naturally from the joint size-speed distribution of the micrometeoroid population impinging on the E-region. For a particle to ionise sufficiently to reach the detection threshold above the cutoff altitude, it must be both large-mass and high-velocity, and because there are so few of these particles, the event rate drops dramatically above the cutoff.

Particles in the sub- $0.05 \mu \mathrm{g}$ mass range are very numerous and expected to contribute a significant fraction of the total meteor mass flux. Unfortunately, our observational evidence suggests that they are not detected by either of the two EISCAT systems. Referring back to Fig. 10, we recall that particles below a critical size $(0.05 \mu \mathrm{g}$ for the UHF, $0.01 \mu \mathrm{g}$ for the VHF) will never approach critical ionization density at any altitude. For these small particles to be detected by radar, the system must be so sensitive that it can detect even the backscatter from centimetre-sized underdense clouds of plasma at $\sim 100 \mathrm{~km}$. While this problem is outside the core topic of the present paper, it should be noted that of all the world's HPLA radar systems, only Arecibo (and possibly Jicamarca) are likely to be sensitive enough to do this. All others have receiving antennas with collecting areas about $-15 \ldots-20 \mathrm{~dB}$ below the Arecibo and Jicamarca ones and so are likely to require the onset of a strong scattering mode to make the meteor-produced plasma visible. This suggests that one has to use caution when using the HPLA radar method for meteor mass flux determinations; for any radar, a part of the low mass end of the true meteoroid population will go undetected, the exact amount being determined by the sensitivity limit of the particular system.

\section{Conclusions}

In this paper we have presented the observation by EISCAT UHF and VHF radars of a meteor head echo cutoff effect, reminiscent of the trail echo ceiling effect observed by conventional normal incidence meteor radars. However, as the radar cross sections at the cutoff height, deduced from our head echo observations, correspond to target diameters significantly smaller than the classical meteor radar trail diameters, the processes causing the cutoff effects cannot be the same.

Measurements of the head echo ceiling heights performed during two different seasons (August and December) show that, while the cutoff height varies with season, it occurs at a constant atmospheric mean free path value for a given wavelength; the height variations only reflect the seasonal variations in atmospheric pressure distribution. The cutoff height also depends on the probing wavelength, but the dependence is nonlinear and cannot be explained by the initial radius effect.

It is shown that, for meteors interacting with the atmosphere in the free molecular regime, the observed target sizes can be interpreted as small concentrations of plasma being generated by single collisions and contained through secondary collisions in a small hemispheric region in front of the meteoroid. The resulting plasma densities are found to be high enough to exceed the critical densities at $224 \mathrm{MHz}$ and, farther down in the atmosphere, also at $931 \mathrm{MHz}$, so that overdense Rayleigh scattering can be invoked as a possible scattering mechanism. In this picture, the very abrupt upper cutoffs in the altitude distributions can be understood simply as a consequence of the joint size-speed distribution of the micrometeoroid population impinging on the E-region.

The observability of low mass meteors is discussed. It is concluded that all HPLA radars are likely to miss a part of the low mass end of the particle size distribution, that the loss is likely to be significant at most EISCAT-class systems and that caution should be exercised when using HPLA meteor data for meteor mass flux determinations.

Acknowledgements. We thank E. Murad for valuable comments. We gratefully acknowledge the EISCAT staff for their assistance during the experiment. The EISCAT Scientific Association is supported by the Centre National de la Recherche Scientifique of France, the Max-Planck-Gesellschaft of Germany, the Particle Physics and Astronomy Research Council of the United Kingdom, Norges Almenvitenskapelige Forskningsråd of Norway, Vetenskapsrådet of Sweden, Suomen Akatemia of Finland, and the National Institute of Polar Research of Japan. The work of one of the authors (A. Pellinen-Wannberg) is supported by the European Office of Aerospace Research and Development, Air Force Office of Scientific Research, Air Force Research Laboratory, under Contract No. F61775-01-WE084.

Topical Editor U.-P. Hoppe thanks A. Webster and D. Janches for their help in evaluating this paper. 


\section{References}

Bronshten, V. A.: Physics of meteoric phenomena, D. Reidel publishing company, 1983.

Ceplecha, Z., Borovicka, J., Elford, W. G., Revelle, D. O., Hawkes, R. L., Porubcan, V., and Simek, M.: Meteor phenomena and bodies, Space Sci. Rev., 84, 327-471, 1998.

Close, S., Oppenheim, M. M., Hunt, S., and Duryd, L.: Scattering characteristics of high-resolution meteor head echoes detected at multiple frequencies, J. Geophys. Res., 107(A10), doi:10.1029/2002JA009253, 2002.

Erickson, P., Lind, F. D., Wendelken, S. M., and Faubert, M. A.: Meteor head echo observations using the Millstone Hill UHF incoherent scatter radar system, in Proceedings of the Meteoroids 2001 Conference, vol. ESA SP-495, pp. 457-463, Swedish Institute of Space Physics, Kiruna, Sweden, 2001.

Hedin, A. E.: Extension of the MSIS thermosphere model into the middle and lower atmosphere, J. Geophys. Res., 96, 1159-1172, 1991.

Hey, J. S., Parsons, S. J., and Stewart, G. S.: Radar observations of the Giacobinid meteor shower, Mon. Not. R. Astron. Soc, 107, 176, 1947.

Hughes, D. W.: Cosmic Dust, John Wiley, New York, 1978.

Hunt, S., Close, S., Oppenheim, M., and Duryd, L.: Twofrequency meteor observations using the Advanced Research Project Agency Long Range Tracking and Instrumentation Radar (ALTAIR), in Proceedings of the Meteoroids 2001 Conference, vol. ESA SP-495, pp. 451-455, Swedish Institute of Space Physics, Kiruna, Sweden, 2001.

Janches, D., Mathews, J. D., Meisel, D. D., and Zhou, Q.: Micrometeor observations using the arecibo $430 \mathrm{MHz}$ radar: I. Determination of the ballistic parameters from measured Doppler velocity and deceleration results, Icarus, 145, 53-63, 2000.

Janches, D., Meisel, D. D., and Mathews, J. D.: Orbital properties of the Arecibo micrometeors and Earth interception, Icarus, 150, 206-218, 2001.
Janches, D. A., Pellinen-Wannberg, A., Wannberg, G., Westman, A., Häggström, I., and Meisel, D. D.: Tristatic observations of meteors using the $930 \mathrm{MHz}$ European Incoherent scatter radar system, J. Geophys. Res., 107(A11), doi:10.1029/2001JA009205, 2002.

Jones, J. and Webster, A. R.: Visual and radar studies of meteor head echoes, Planet. Space Sci., 39, 873-878, 1991.

Love, S. G. and Brownlee, A : Direct measurement of the terrestrial mass accretion rate of cosmic dust, Science, 262, 550-553, 1993.

Manning, L. A.: The initial radius of meteoric ionization trails, J. Geophys. Res., pp. 181-196, 1958.

Mathews, J. D., Meisel, D. D., Hunter, K. P., Getman, V. S., and Zhou, Q.: Very high resolution studies of micrometeors using the Arecibo $430 \mathrm{MHz}$ radar, Icarus, 126, 157-169, 1997.

McKinley, D. W. R.: Meteor science and engineering, McGrawHill, 1961.

Olsson-Steel, D. and Elford, W. G.: The height distribution of radio meteors: observations at $2 \mathrm{MHz}$, J. Atmos. Terr. Phys., 49, 243 258, 1987.

Pellinen-Wannberg, A. and Wannberg, G.: Meteor observations with the European incoherent scatter UHF radar, J. Geophys. Res., 99, 11379-11390, 1994.

Pellinen-Wannberg, A., Westman, A., Wannberg, G., and Kaila, K. Meteor fluxes and visual magnitudes from EISCAT radar event rates: A comparison with cross section based magnitude estimates and optical data, Ann. Geophys., 16, 1475-1485, 1998.

Steel, D. I. and Elford, W. G.: The height distribution of radio meteors: comparison of observations at different frequencies on the basis of standard echo theory, J. Atmos. Terr. Phys., 53, 409-417, 1991.

Wannberg, G., Pellinen-Wannberg, A., and Westman, A.: An ambiguity-function-based method for analysis of Doppler decompressed radar signals to EISCAT measurements of oblique UHF-VHF meteor echoes, Radio sci., 31, 497-518, 1996.

Zhou, Q. and Kelley, M. C.: Meteor observations by the arecibo $430 \mathrm{MHz}$ incoherent scatter radar: II. Results from time-resolved observations, J. Atmos. Terr. Phys., 59, 739-752, 1997. 